“ (C) 2015 IEEE. Personal use of this material is permitted. Permission from IEEE must be obtained for all other uses, in any current or future media, including

reprinting/republishing this material for advertising or promotional purposes, creating new collective works, for resale or redistribution to servers or lists, or reuse of any copyrighted component of this work in other works." 


\title{
Blue Pages: Software as a Service Data Set
}

\author{
Asma Musabah Alkalbani*,Ahmed Mohamed Ghamry ${ }^{\dagger}$, Farookh Khadeer Hussain*,Omar Khadeer Hussain ${ }^{\dagger}$ \\ Decision Support and e-Service Intelligence Lab* \\ Quantum Computation and Intelligent Systems \\ School of Software, University of Technology \\ Sydney, NSW 2007, Australia \\ Email: Asma.M.Alkalbani@student.uts.edu.au, Farookh.Hussain@uts.edu.au \\ School of Business ${ }^{\dagger}$ \\ University of New South Wales Canberra (UNSW Canberra) \\ Australian Defence Force Academy \\ Canberra, ACT, 2602 \\ Email:a.ghamry@unsw.edu.au,O.Hussain@adfa.edu.au
}

\begin{abstract}
Blue Pages is a repository that contains data collected on Software as a Service(SaaS).The repository lists SaaS information according to an organization's functional needs. Blue Pages holds thousands of records on SaaS applications' business profiles. The data was extracted between February 2015 and August 2015 from the following web portals: www.cloudreviews.com \& www.getApp.com. Every record holds details on the service offerings, including service name, the date the service was founded, service category, free trial (yes/no), mobile app (yes/no), starting price, service description, service type and provider link. The dataset is the first to provide information on SaaS.The dataset presented in this paper can be used in future research in cloud service selection and cloud service discovery. The dataset is available online through the Blue Page website http://www.bluepagesdataset.com/.
\end{abstract}

Keywords-Cloud services, SaaS, Service Discovery, SaaS dataset, web harvesting.

\section{INTRODUCTION}

Cloud computing resources play a critical role in IT cost reduction, scalability and collaboration efficiency for business organizations by offering IT resources on-demand with marketplace cloud services classified in terms of the computing resources being provided, namely Software as a Service (SaaS), PaaS (Platform as a Service), IaaS (Infrastructure as a Service)[5]. Recently, there has been growing interest in the industry to adopt SaaS since it guarantees benefit through cost saving and increased productivity[3]. Figure 1 illustrates a comparative analysis, reported by Cisco, between different types of adopted cloud services, showing that SaaS applications will be the most highly deployed global cloud service by 2018.

With the increased adoption of SaaS, the amount of SaaS applications and service providers is extensive and unmanageable on the World Wide Web (WWW)[8]. Currently, the main major resource for discovering cloud services is web search engines such as Bing, Google, and Yahoo!. These search engines are useful for searching for cloud services, however, these engines are only designed to locate and point to the URLs of service offerings[6]. They can only do part of the work; the rest has to be done by consumers, such as scanning the content of each website to find the best service offerings, and comparing the various service offerings. Also comparing service offerings, in terms of quality and price, is another concern of consumers. Web directories and web portals, such

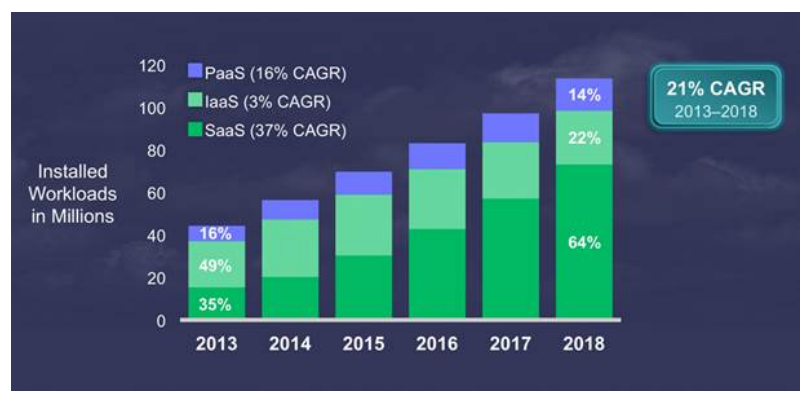

Fig. 1: SaaS most highly deployed cloud service by 2018

as www.getApp.com and www.cloudreviews.com, are other methods of collecting details on cloud service offerings and obtaining a list of matching cloud services[1][2]. For some of these websites, it is difficult to keep up with daily changes, such as fluctuations in price, while others, such as getApp continually update their service list. In the cloud marketplace, the main challenge faced by many researchers and consumers is the lack of a central cloud repository that provides a complete listing of the cloud services available in the market, where the end users can easily compare and select the most appropriate services.

Given the increasing use of SaaS in a number of disciplines, such as education, business, health, a central repository would be beneficial for both consumers and providers[4],[8]. The SaaS marketplace project aimed to investigate SaaS applications and providers on the World Wide Web (WWW). The objective of the project is to offer a real world SaaS offerings dataset called the Blue Page Dataset for future research. This dataset provides sufficient and complete information about each SaaS application offering.

To achieve our objective, we used a web harvesting technique. Web harvesting (web scraping) is a very well-known tool that offers solutions for searching and gathering web information[6]. It focuses on extracting unstructured informa- 
tion from pages and data on the WWW. It complements the use of a search engine; the search engine usually locates the information web page and the web harvesting tool does the rest, such as organizing and gathering unstructured information from the web page source. For the purpose of this study, we develop a web scraper to collect details on SaaS offerings from web portals. After conducting a survey of several web portals, we found that there are two excellent resources for information on SaaS offerings namely: www.getApp.com, and www.cloudrewviews.com.

We use the web scraping tool to collect meta descriptions of SaaS offerings from the web portals previously mentioned. The meta descriptions are used to extract specific content and meta keywords embedded in the web pages, such as information on SaaS offerings, and then build the SaaS dataset. The dataset contains the following information: service name, when the service was founded, service category, free trial(yes/no), mobile app(yes/no), starting price, service description, service type and provider link. At this stage of the project, we only harvest two portals, but we plan to harvest more web directories and portals in the future and provide an automated or semi-automated updating technique to cope with daily changes in the service offering in the cloud marketplace.

At this stage 1, the dataset contains more than five thousand details on real SaaS offerings harvested from the two well-known cloud web portals, previously mentioned. Additionally, based on the information collected on the SaaS applications, statistical analyses were performed to present the current status of SaaS applications and their providers in the marketplace.

The rest of the paper is organized as follows: Section 2 describes the problems with existing datasets. Section 3 discusses the methodology for collecting the dataset. Section 4 describes the schema of the datasets. Concluding remarks are discussed in Section 5. Finally, the conclusion and future work are discussed in Section 6.

\section{PROBLEMS WITH EXISTING DATASETS}

According to the literature, there is only one study that presents a cloud services dataset [7]. Talal et al. presented three datasets, namely: the Main Cloud Service dataset, the WSD \& WADL dataset, and the Trust Feedback dataset. The Main Cloud Service dataset only provides a list of cloud service URLs with no further details, whereas the WSDL \& WADL dataset provides a list of cloud services along with a service description language (WSDL). The Trust Feedback dataset presents the feedback of consumers on the Quality of Service (QoS) of the cloud service. This dataset includes consumer name and QoS feedback attributes, such as availability and price. Talal found that the majority of cloud providers provide IaaS (52.29\%) compared with SaaS $(27.08 \%)$ and PaaS (7.70\%). However, this dataset does not provide sufficient information, for instance the customer name data value is " $2 \mathrm{~d} 70 \mathrm{~d} 7269 \mathrm{c} 514 \mathrm{da} 2 . .$. ", and service name data value is "land1", and there is also a lack of corresponding service description and service type information. Furthermore, there is no mechanism by which the existing repository can be automated or updated, based on change to service offerings.

\section{METHODOLOGY}

Our methodology comprises three phases: metadata collection, web scraper tool, and repository. In the rest of this section, the details of each phase are given.

Phase1. Metadata collection: The aim of this phase is to collect metadata on SaaS offers. We target two web portals: cloudreviews and getApp, which are excellent resources for SaaS offerings. We downloaded the whole SaaS offerings page source (metadata description). Then, the meta data description is stored in a local SQL database for further analysis.

Phase 2. Web scraper tool: In this phase, we develop a scraper tool to automatically extract information on SaaS offerings from the metadata description, downloaded in (Phase 1). We scraped specific meta tags from the SaaS offerings meta description to collect information on the SaaS offerings, such as (\& raquo; Free Trial $<$ span $>$ Yes)

Phase 3. Repository: In this phase, we build an SQL database to store the descriptions extracted on the SaaS offerings, obtained in (Phase 2). The database schema is discussed in detail in the next section.

\section{The software technical specifications are:}

- Platform: Net Framework 4.0 on Visual studio 2010.

- $\quad$ Web Server: IIS 6.0.

- Programming language: ASP.Net C.

- $\quad$ Database: SQL Server 2008 R2.

\section{DATASET DESCRIPTION}

This intent of the Blue Pages dataset is to provide a complete dataset of SaaS offerings for consumers and researchers. Currently, the dataset has 5294 records and it consists of 5 main items, which are described as follows:

Database Item 1 (Table: MD Service). This item contains all the descriptions of the SaaS offering that were extracted from web portals, namely cloudreviews, and getApp. The attribute ' $I d$ ' represents the code for the SaaS service used in the Blue Page project. The ' $I d$ ' number is a unique number which is generated automatically for each service in the database. The 'StrServiceName' represents the name of the service, for example, the Desk.com application from Sales force. The attribute 'StrFounded' represents the service establishment date, for example, Desk.com was established in 2009. The attribute 'StrCategory' represents the type of service, such as data analytics or customer support. The attribute 'StrFreeTrial' denotes if there is a free trial available for this service. The attribute 'StrEditorRating' refers to the rating of the service by the end-user. The attribute 'StrStartingPrice' represents the starting price of the service according to the marketplace. The attribute 'StrBriefDescription' represents a brief description of the application, including the standard features of the service. The attribute 'StrServiceType' denotes the category of the service (for example a data analytics application belongs to the Business Intelligence group, and customer support belongs to the CRM. Figure 2. shows the schema of MD Service.

Database Item 2 (Table: Service Review) This table contains a listing of SaaS consumer reviews and feedback on 


$\begin{array}{lll}\vdots \text { MD_Service } & \\ \vdots & \text { Id } & \vdots \\ & \text { StrServiceName } & \vdots \\ & \text { StrFounded } & \vdots \\ & \text { StrCategory } & \vdots \\ & \text { StrFreeTrial } & \vdots \\ & \text { StrMobileApp } & \vdots \\ & \text { StrEditorRating } & \vdots \\ & \text { StrStartingPrice } & \vdots \\ & \text { StrBriefDescription } & \vdots \\ & \text { StrServiceType } & \vdots \\ & \text { StrProviderlinks } & \vdots \\ \text { DTDateInserted } & \vdots\end{array}$

Fig. 2: Schema of the table for collected service

\begin{tabular}{|c|}
\hline Service_Review \\
\hline$P K$ Int Id \\
\hline Str_Review_Date \\
\hline Str_Reviewer_Name \\
\hline Int_Service_Id \\
\hline Str_Review \\
\hline
\end{tabular}

Fig. 3: Schema of the table for service review

the service. Figure 3. illustrates schema of Service Review table. The attribute 'Str Review Date' lists the date of the review while the attribute 'Str Reviewer Namee' stores the name of the reviews and 'Str Review' presents the consumers' reviews on each SaaS.

Data Model: Figure 4 illustrates the data model of our dataset. The dataset consists of two tables: 'MD Service' and 'Service Review'. The former contains all the attributes of the SaaS offering descriptions, while the latter stores the service review information. As shown in the figure below, the relationship between the tables is 1-m multiple reviews for one service. Existing publicly available datasets only include the 'MD Service' while the 'Service Review' omitted from the dataset due to future research. Table 1 presents the sample of data in the 'Service Review' which will be released online in the second version of the Blue Pages Data Set.

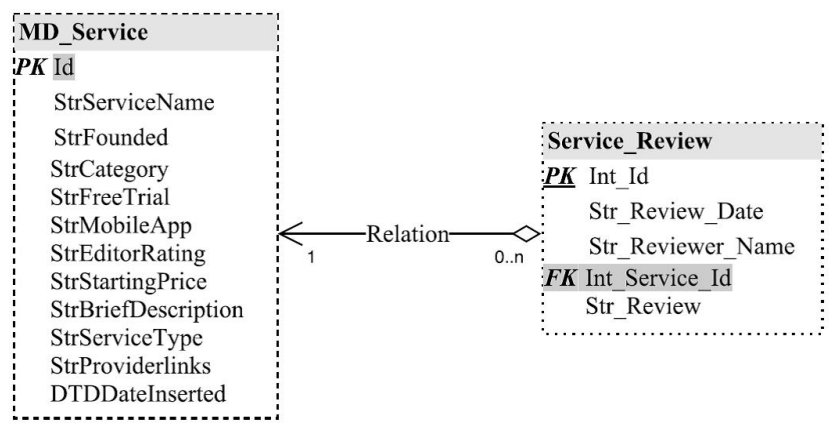

Fig. 4: Schema of the table for collected service as needed to properly center the text within the cells

TABLE I: Service Review Sample Data

\begin{tabular}{|c|c|c|c|c|}
\hline ID & $\begin{array}{l}\text { Review } \\
\text { Date }\end{array}$ & $\begin{array}{l}\text { Reviewer } \\
\text { Name }\end{array}$ & $\begin{array}{l}\text { Service } \\
\text { ID }\end{array}$ & Review \\
\hline 1 & $\begin{array}{l}7 \text { th } \\
\text { Septem- } \\
\text { ber,2010 } \\
05: 34\end{array}$ & Jason $\mathrm{H}$ & 154 & $\begin{array}{l}\text { super useful tool } \\
\text { with great features }\end{array}$ \\
\hline 2 & $\begin{array}{l}\text { 28th } \\
\text { March, } \\
2010 \\
06: 35\end{array}$ & KWilliams & 155 & $\begin{array}{l}\text { The guys at price } \\
\text { grid are amazing. } \\
\text { We required a } \\
\text { custom solution } \\
\text { to meet some of } \\
\text { our goals. It was } \\
\text { delivered quickly, } \\
\text { with no effort on } \\
\text { our end other then a } \\
\text { few go to meeting's }\end{array}$ \\
\hline 3 & $\begin{array}{l}31 \text { st } \\
\text { August } \\
16: 59\end{array}$ & $\begin{array}{l}\text { Lee } \\
\text { Schneider }\end{array}$ & 160 & $\begin{array}{l}\text { If you really } \\
\text { want to cover } \\
\text { your bases, use } \\
\text { Mention to monitor } \\
\text { your clients, } \\
\text { your competitors, } \\
\text { and important } \\
\text { keywords. There } \\
\text { is no other app } \\
\text { that tracks the web } \\
\text { more completely. } \\
\text { The reporting and } \\
\text { status are great. }\end{array}$ \\
\hline
\end{tabular}

\section{RESULTS AND ANALYSIS}

An initial objective of our study is to develop a Blue Pages dataset and to provide to end users and research communities a complete and sufficient SaaS offerings dataset. This study presents the first version of the Blue Pages dataset, where there are currently 5294 items of service information collected from getApp and cloudreviews. Further, the analysis indicates the some emerging trends of SaaS applications in the marketplace, which is discussed in detail in the next section.

\section{A. SaaS Classification}

An interesting finding from the Blue Pages Data Set is that the SaaS applications are classified on the basis of organizational function, such as HRM, business intelligence, communication, business intelligence analytics, sales, HR employee management, customer management, collaboration applications and operations management. It can be seen from the data in (Figure.5) that the majority of SaaS applications are for management and collaboration, while a small number of applications are available for human resources, business intelligence and communication.

\section{B. SaaS Mobile Application}

Another important finding is that the result indicates that of the 5294 applications, only 1348 were reported as mobile applications. Figure 6 shows that $25 \%$ of the services offered are mobile applications.

\section{CONCLUDING REMARKS}

In our study, we investigated the distribution of SaaS applications in the WWW. The results show that there are 5294 SaaS applications offered on two well-known web portals: getApp and cloudreviews. Some initial insights were presented 


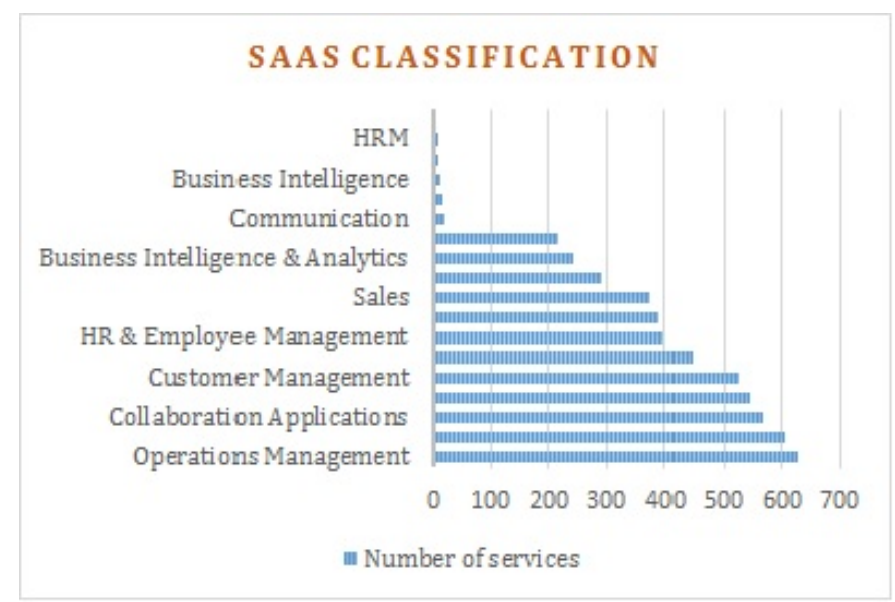

Fig. 5: SaaS Classification from Blue Pages Data Set

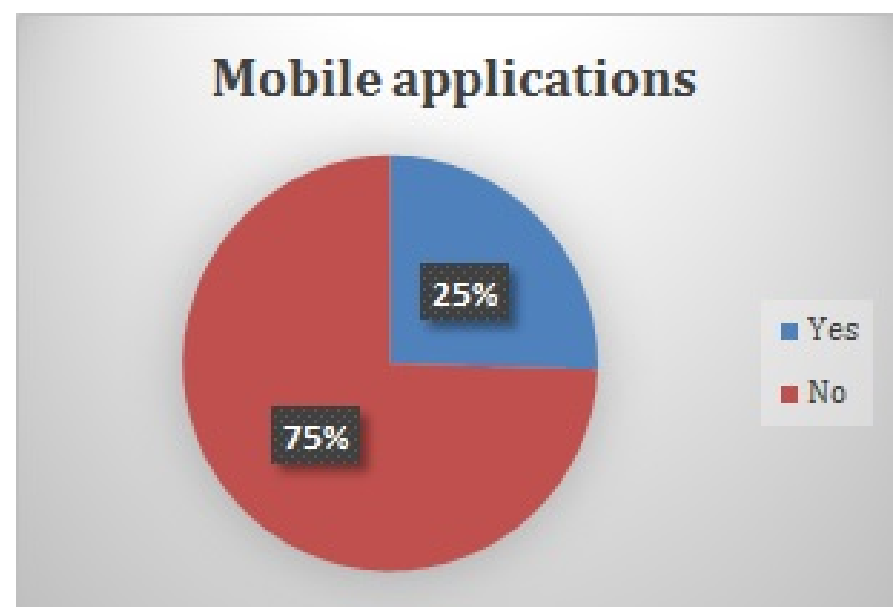

Fig. 6: Number of SaaS Mobile Application

as well. An interesting finding is that the SaaS applications are classified according to the organisation's functional needs. Such service statistics may assist market developers and the research community to examine the status of the current market and provide better SaaS applications and SaaS discovery solutions to discover SaaS solutions. Although UDDI is an existing approach for finding web services, for SaaS, it is unclear whether the market needs such a central repository or whether decentralized review portals will replace it.

\section{DATASET AVAILABILITY}

The dataset discussed in this paper is publicly available at http://www.bluepagesdataset.com/

\section{REFERENCES}

[1] Cloud reviews. http://www.cloudreviews.com/. Accessed Sept 10, 2015.

[2] Getapp. https://www.getapp.com/. Accessed Sept 10, 2015.

[3] Reduce costs and increase performance: The saas advantage for contact centers. http://www.incontact.com/sites/default/files/resources/ saas-contact-center-software.pdf. Accessed Sept 10, 2015.

[4] The evolution of cloud adoption in australia. https://www.melbourneit com.au/ng/downloads/es-resources/Cloud_Adoption_Whitepaper.pdf. Accessed Sept 10, 2015.
[5] F. Liu, J. Tong, J. Mao, R. Bohn, J. Messina, L. Badger, and D. Leaf. Nist cloud computing reference architecture. NIST special publication, 500:292, 2011.

[6] J. Marill, A. Boyko, and M. Ashenfelder. Web harvesting survey. International Internet Preservation Consortium, 20, 2004.

[7] T. H. Noor, Q. Z. Sheng, A. Alfazi, A. H. Ngu, and J. Law. Csce: a crawler engine for cloud services discovery on the world wide web. In Web Services (ICWS), 2013 IEEE 20th International Conference on, pages 443-450. IEEE, 2013.

[8] A. E. Paul D. Hamerman, Liz Herbert with Christopher Andrews. Application adoption trends 2015: The saas boom continues as businesses demand agility. https://www.forrester.com/Application+ Adoption+Trends+2015+The+SaaS+Boom+Continues+As+Businesses+ Demand+Agility/fulltext/-/E-RES117364. Accessed Sept 10, 2015. 


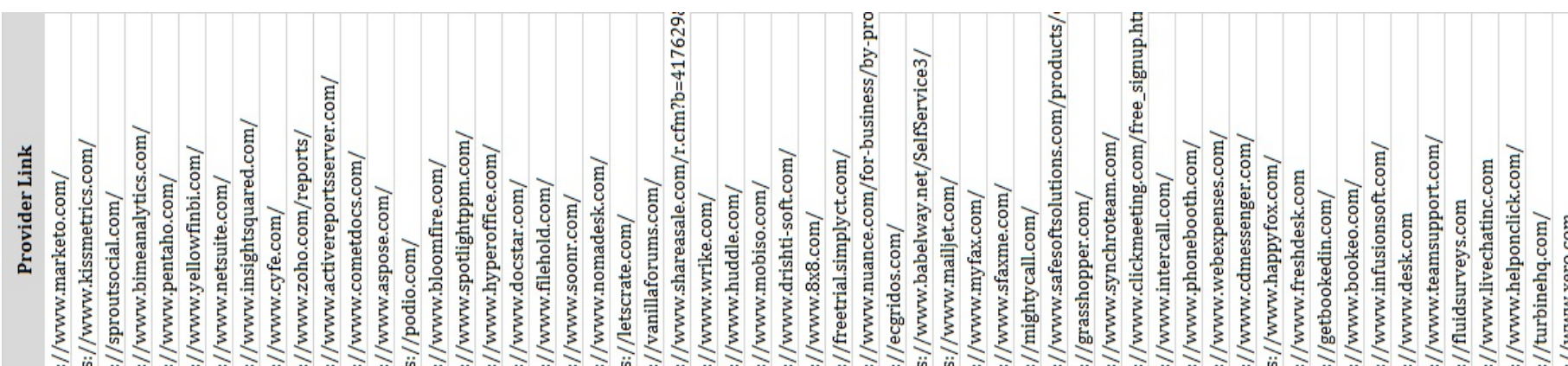

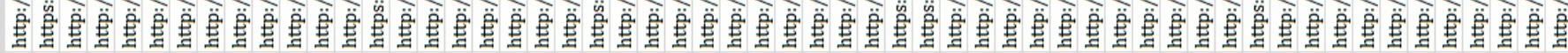

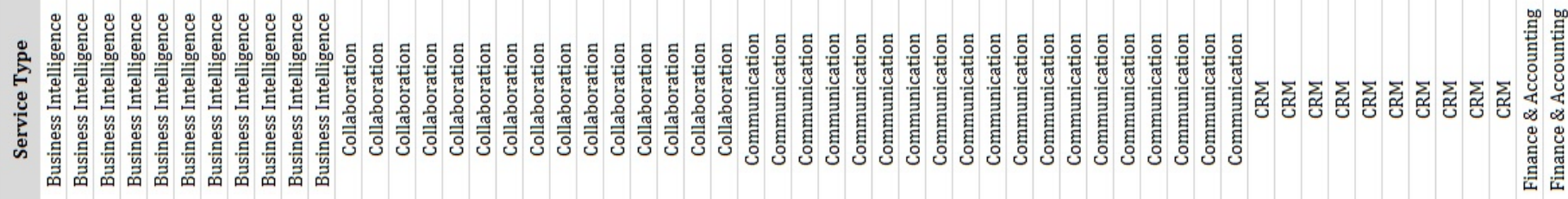

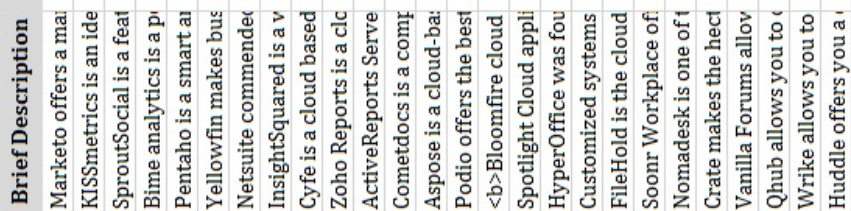

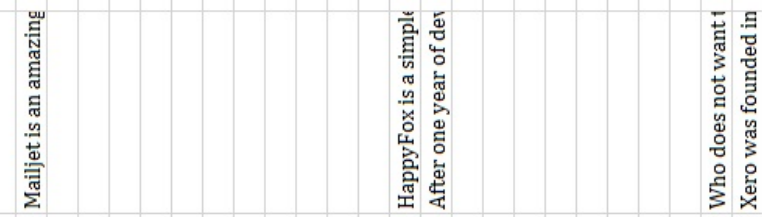

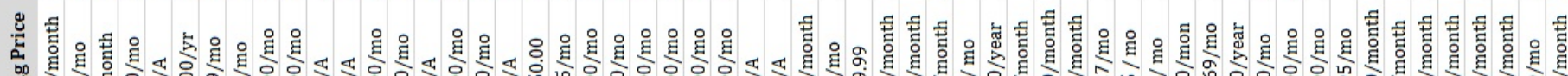

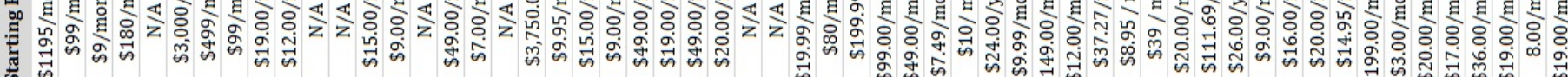

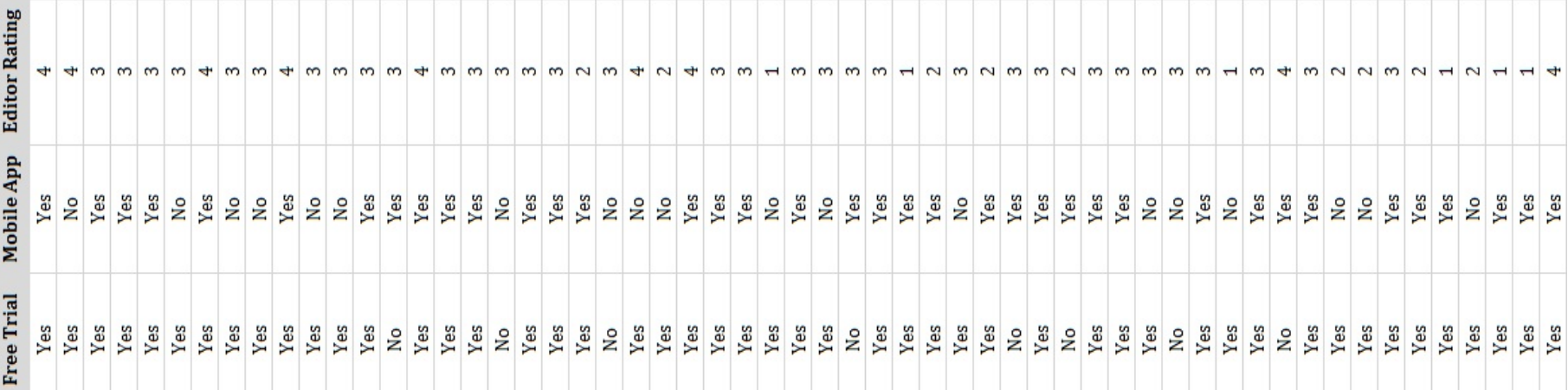
일

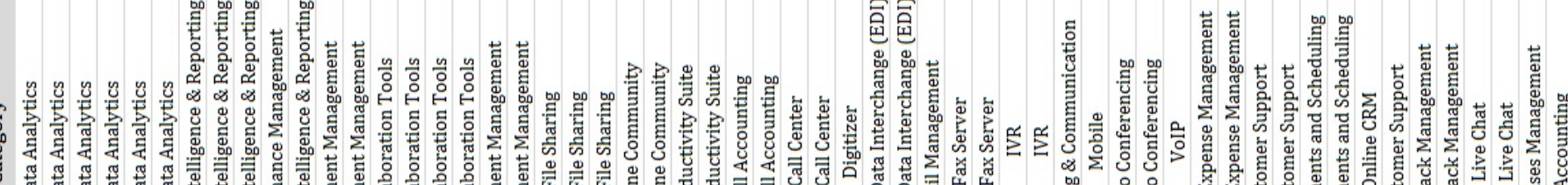

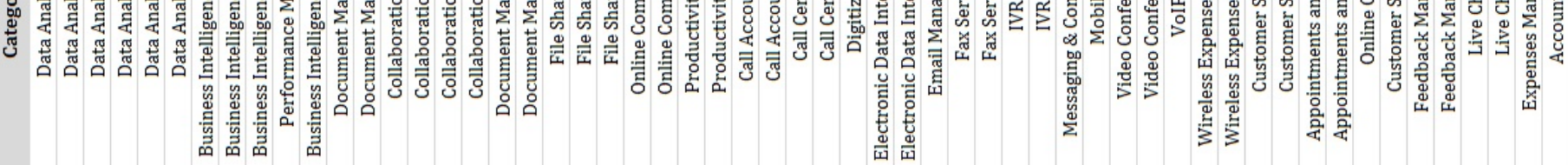

Mn⿴囗十)

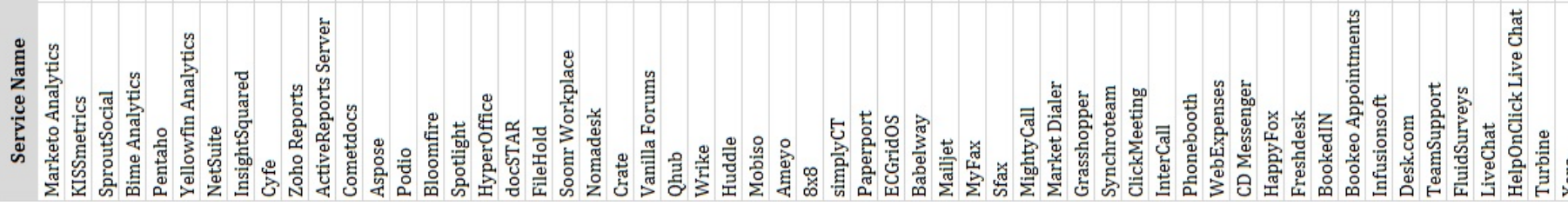
Fig. 7: list of SaaS applications offers 


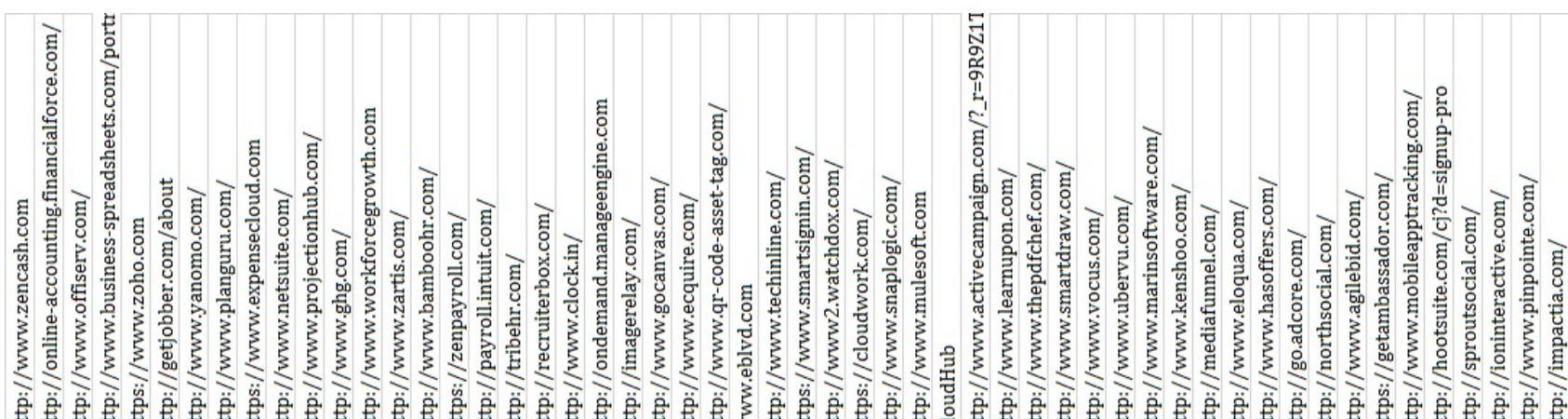

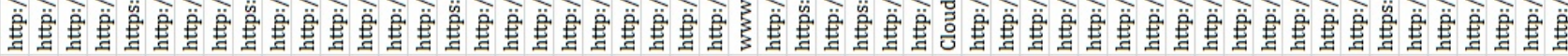

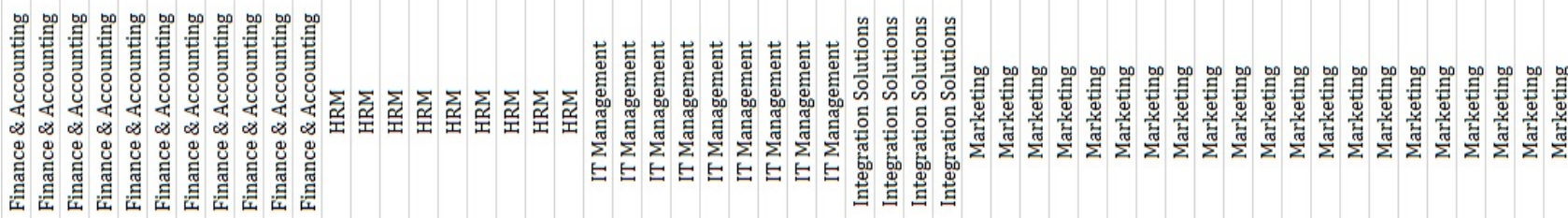

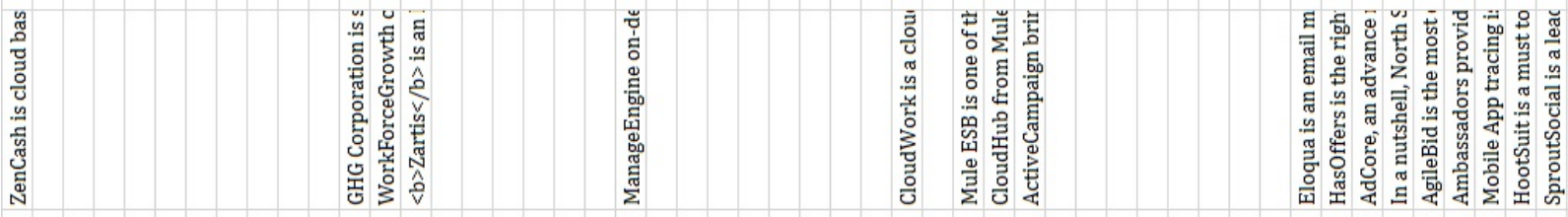

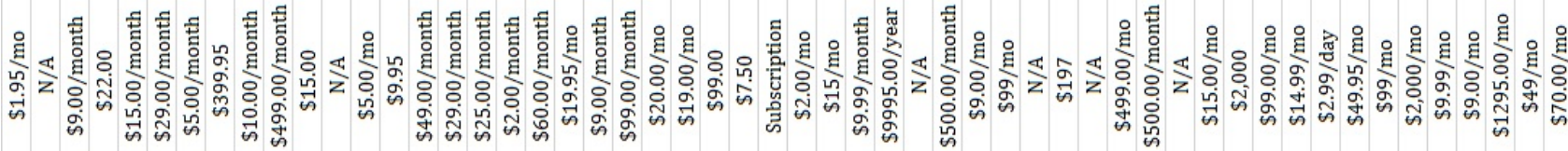

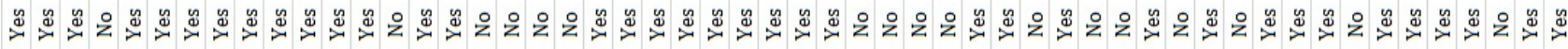

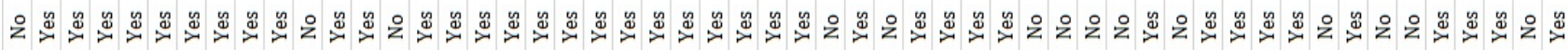

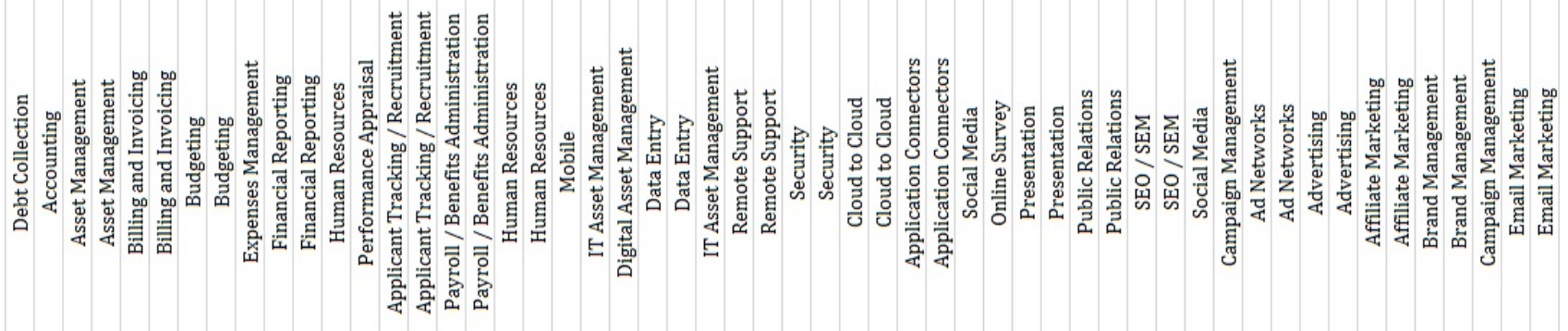

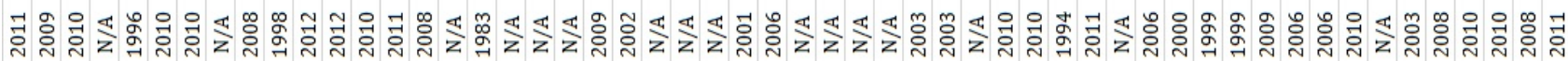

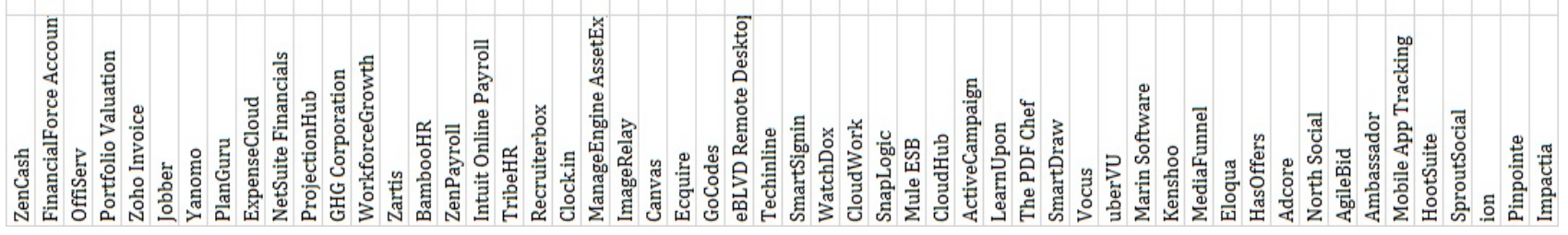

Fig. 8: list of SaaS applications offers 